\title{
Testing the Bell Inequality at Experiments of High Energy Physics
}

\author{
Xi-Qing Hao ${ }^{1}$, Hong-Wei Ke ${ }^{2}$, Yi-Bing Ding ${ }^{3}$, Peng-Nian Shen ${ }^{4}$ and Xue-Qian $\mathrm{Li}^{1}$ \\ ${ }^{1}$ Department of Physics, Nankai University, Tianjin 300071; \\ ${ }^{2}$ Department of Physics, Tianjin University, Tianjin 300072; \\ ${ }^{3}$ Department of Physics, Graduate University of \\ Chinese Academy of Sciences, Beijing 100049, \\ ${ }^{4}$ Institute of High Energy Physics, Beijing 100049
}

(Dated: November 1, 2018)

\begin{abstract}
Besides using the laser beam, it is very tempting to directly testify the Bell inequality at high energy experiments where the spin correlation is exactly what the original Bell inequality investigates. In this work, we follow the proposal raised in literature and use the successive decays $J / \psi \rightarrow \gamma \eta_{c} \rightarrow \Lambda \bar{\Lambda} \rightarrow p \pi^{-} \bar{p} \pi^{+}$to testify the Bell inequality. Our goal is twofold, namely, we first make a Monte-Carlo simulation of the processes based on the quantum field theory (QFT). Since the underlying theory is QFT, it implies that we pre-admit the validity of quantum picture. Even though the QFT is true, we need to find how big the database should be, so that we can clearly show deviations of the correlation from the Bell inequality determined by the local hidden variable theory. There have been some critiques on the proposed method, so in the second part, we suggest some improvements which may help to remedy the ambiguities indicated by the critiques. It may be realized at an updated facility of high energy physics, such as BES III.
\end{abstract}




\section{INTRODUCTION}

Since the birth of Quantum Mechanics (QM), dispute about its essence never ceases. Indeed, nowadays nobody still doubts validity of QM because of its great success in all fields. However, one can ask if the theory of QM is complete and the relevant principles, such as the wave-particle duality, superposition principle and the probability interpretation are fundamental in nature or just effective representation of other underlying principles.

Around what is the essence of QM, Bohr and Einstein conducted a sharp debate over many years. In 1935, Einstein, Podolsky and Rosen published a collaboration paper where they made a challenge to the completeness of QM, and it is the famous EPR paradox [1]. It helps to develop the local hidden variable theory (LHVT), especially Bohm resurrected this field[2], and then based on this new understanding, Bell raised a theorem which proves that a local hidden variable theory cannot repeat all predictions of QM [3]. Because of the local hidden variables, definite correlations of the involved states would be retained, even though they are separated by a space-like distance. By contrast, the quantum entanglement is supposed to be a fundamental character of nature and it manifests the difference of quantum mechanics from classical physics. To search a testable scheme which can distinguish between QM and the LHVT, Bell established the Bell inequality for correlation among spin polarizations of various states[3]. By the Bell inequality, if the correlation is due to a set of local hidden variables, the inequality must hold, however, one can easily show that within a certain parameter range the quantum superposition of different states would spoil this inequality. Explicitly, in literature, it is suggested that correlation of polarizations of two spin-1/2 particles in a singlet state of the total spin, i.e. $|0,0\rangle$, the Bell inequality holds if the mechanism of local hidden variable theory (LHVT) applies, whereas it may be explicitly violated as QM is the dominating mechanism. That is an exclusive and direct test of LHVT. For almost half century, many experimental schemes have been designed to realize

such a measurement [4, 5, 6, 7]. However, it was noticed that such experiments demand high accuracy and statistics, so that become very difficult. Just because of the difficulties, so far even though the importance of the test is obvious, people are still unable to carry out experiments with high precision to make a definite conclusion yet. No wonder, the first success was achieved in optical experiments, where the correlation between photon polarizations is studied thanks to application of high quality laser beams and high precision of 
optical apparatus as well as advanced techniques. Over a half century, many experiments have been carried out to testify, among them, the polarization entanglement experiments of two-photons and multi-photons attract the widest attention of the physics society [6]. All photon experimental data indicate that the Bell inequality and its extension forms are violated, and the results are fully consistent with the prediction of QM. The consistency can reach as high as 30 standard deviations. On other aspect, however, as indicated in literature, the detection efficiency in optical experiments is rather low, therefore when analyzing the data, one needs to introduce additional assumptions, so that the requirement of LHVT cannot be completely satisfied. That is why as generally considered, so far, the Bell inequality has not undergone serious test yet.

It would be interesting to return to the original formulism where correlation between polarizations of two spin-1/2 particles is discussed. However, because of existence of Lorentz force, the spin polarization of a charged fermion cannot be directly measured even though a inhomogenous magnetic field is employed in experiment [8] (we will discuss this problem again at the end of this paper). Some authors, alternatively suggested to directly measure the polarization correlation at experiments of high energy physics [4]. An ideal candidate is the successive decay mode of $\eta_{c} \rightarrow \Lambda \bar{\Lambda} \rightarrow p \pi^{-}+\bar{p} \pi^{+}$, because $\eta_{c}$ is a $0^{-+}$meson and thus $\Lambda \bar{\Lambda}$ would be in the spin $\mid 0,0>$ state. That is an entangled state of two spin- $1 / 2$ fermions. An obvious advantage is that the information of the spin polarization of $\Lambda(\bar{\Lambda})$ can be obtained via measuring the directions of the emitted $\pi^{-}\left(\pi^{+}\right)$, which would leave clear tracks in the detector.

In the original proposal for testing the Bell inequality, there should be three independent directions and the polarizations along all the three directions must be measured and the formulation is

$$
\left|E(\mathbf{a}, \mathbf{b})-E\left(\mathbf{a}, \mathbf{b}^{\prime} \mid\right)\right| \leqslant 1+E\left(\mathbf{b}, \mathbf{b}^{\prime}\right)
$$

where $E\left(\mathbf{n}_{\mathbf{1}}, \mathbf{n}_{\mathbf{2}}\right)$ is the correlation of two polarizations of the two particles which exist in an entangled state and separated by a space-like distance. As suggested in the original literature, the inequality with three independent directions is reduced into a form with only one continuous parameter as

$$
E\left(\theta_{a b}\right) \leqslant 1-\theta_{a b} / \pi
$$

where $\theta_{a b}$ is the angle between the linear momenta of the two emitted pions in the respective 
CM frames of $\Lambda$ and $\bar{\Lambda}$. The authors of Ref. [4] showed that the QM result would upset this inequality. Obviously, it is a very difficult experiment which demands a precise measurement. Thanks to the improvements of detection facility and technique, experimentalists may be able to measure decay widths of small-probability processes. Moreover, the BES possesses a largest database of $J / \psi$ which provides a possibility to test the Bell inequality at high energy experiments.

On another aspect, this scheme has received some critiques. We will try to remedy those problems in the last section of the paper.

In this work, it is worth noticing, we simulate the successive processes $J / \psi \rightarrow \eta_{c}+\gamma$ and $\eta_{c} \rightarrow \Lambda \bar{\Lambda} \rightarrow p \pi^{-}+\bar{p} \pi^{+}$based on the quantum field theory and the aim of such a work is to check if the inequality (2) could be satisfied. A natural question would be raised that the Bell inequality is to testify which one of the local hidden variable theory and QM is valid, thus when we employ the quantum field theory, it implicitly suggests that we have already assumed quantum mechanics applying, so that it is not a real test of the Bell inequality and cannot determine if the local hidden variable theory fails. In fact, in the first part of this work, we are not intending to eventually make final conclusion about the inequality, but will determine how big the database must be to guarantee the precision for drawing a definite conclusion about difference between the predictions of QM and local hidden-variable theory. Since QM is based on statistics, namely all possible quantum states have certain probabilities to occur, we can only obtain the results with a certain statistics. Of course, if the event number can be infinity, the QM would predict a smooth curve for the required relation (the polarization correlation), but definitely it is impossible, thus a question emerges. Namely, if the QM is right and the quantum field theory is a valid theory, we need the number of events to find a clear distinction above the bound set by the Bell inequality with one, two or three standard deviations. We use the Monte Carlo method to simulate the successive processes and draw the graphs with error bars being explicitly marked out. Finally, we find that the necessary number of $\mathrm{J} / \psi$ must be as large as $10^{9}$ and when the statistical and systematic errors of concrete experiments are taken into account, the database must be enlarged at least by a factor of 10 .

Considering the critiques to this method, we propose an improvement scheme. A long time ago, the authors of Ref. [5] suggested to set two Stern-Gerlach apparatuses which provide inhomogenous magnetic fields to carry out an experiment with two proton beams to test 
the Bell inequality. In this work, following their suggestions, we would set such apparatuses which can distinguish different spin polarizations of $\Lambda(\bar{\Lambda})$, namely, due to their anomalous magnetic moments, they undergo different forces and decline their original trajectories. Our sensitive detector can record their decay daughters' trajectories to determine their polarizations. Because we have freedom to adjust the directions of the magnetic fields, we can have three real independent directions as the original Bell inequality requires. A detailed discussion will be given in the last section.

This paper is organized as follows. After this long introduction, we will present all formulae which are necessary for numerical computations and for readers' convenience, we include a subsection to discuss the Monte-Carlo simulation and $\chi^{2}$ analysis. The third section is for our numerical results where the Monte-Carlo errors are given. In section IV, we discuss our proposal which suggests a modified version for probing the Bell inequality, and its advantages and difficulties.

\section{THE BELL INEQUALITY}

As discussed in the introduction, one of the focuses of basic research of modern physics is to directly testify the Bell inequality which is derived based on the LHVT of quantum mechanics, and it is crucial to justify if QM is a complete local theory[9]. Starting from the LHVT of quantum mechanics, one can derive an inequality about the correlation between polarizations of two spin-1/2 fermions in a system of total-spin singlet, i.e. the Bell inequality. In the LHVT with the hidden variable being $\lambda$, the Bell inequality can be phased as

$$
E(\mathbf{a}, \mathbf{b})=\int d \lambda A(\mathbf{a}, \lambda) B(\mathbf{b}, \lambda) \rho(\lambda),
$$

where $\rho(\lambda)$ is a distribution of the hidden variable(s) $\lambda, A(\mathbf{a}, \lambda)$ is the result of measurement of the projection $\left(\boldsymbol{\sigma}^{(A)} \cdot \mathbf{a}\right)$ of spin $\boldsymbol{\sigma}^{(A)}$ of particle A along direction a, whereas $B(\mathbf{b}, \lambda)$ is the corresponding measurement for the projection $\left(\boldsymbol{\sigma}^{(B)} \cdot \mathbf{b}\right)$ of spin $\boldsymbol{\sigma}^{(B)}$ of another particle $\mathrm{B}$ along direction $\mathbf{b}$. The original expression of Bell inequality refers to three independent spatial directions $\mathbf{a}, \mathbf{b}$ and $\mathbf{c}$ as

$$
|E(\mathbf{a}, \mathbf{b})-E(\mathbf{a}, \mathbf{c})|-E(\mathbf{b}, \mathbf{c}) \leqslant 1
$$

According to the theory of QM, this correlation function is the average value of operator 
$\left(\boldsymbol{\sigma}^{(A)} \cdot \mathbf{a}\right) \cdot\left(\boldsymbol{\sigma}^{(B)} \cdot \mathbf{b}\right)$ over a spin singlet $|\psi\rangle\left(\right.$ or written as $\left.\left|\chi_{00}\right\rangle\right)$. Namely:

$$
\begin{aligned}
E(\mathbf{a}, \mathbf{b})_{\mathrm{QM}} & =\left\langle\psi\left|\left(\boldsymbol{\sigma}^{(A)} \cdot \mathbf{a}\right)\left(\boldsymbol{\sigma}^{(B)} \cdot \mathbf{b}\right)\right| \psi\right\rangle \\
& =-\mathbf{a} \cdot \mathbf{b} .
\end{aligned}
$$

It is easy to prove that the Bell inequality (41) contradicts to the QM result. If the QM result receives experimental support, one would quantitatively confirm that the local hidden variable theory cannot describe all predictions of quantum mechanics.

Besides the optical experiments, in recent years, some proposals of non-optical experiments have been raised to testify the LHVT of QM. Similar tests have also been realized at the proton-proton double-scattering [5] and other collider experiments [7], and their conclusions all support the QM and suggest that the Bell inequality is violated. Comparing with optical experiments, high energy experiments are dealing with massive particles, especially the real spin-1/2 fermions. In such processes weak and strong interactions are involved while only EM interaction applies in optical experiments.

Among the proposals, we are especially interested in the successive decay processes $\eta_{c} \rightarrow$ $\Lambda \bar{\Lambda} \rightarrow p \pi^{-} \bar{p} \pi^{+}$at $e^{+} e^{-}$colliders, which was suggested by Törnqvist [4] a long while ago. The reason of our interests is not only because it provides a clear and realizable method, but also the Beijing Electron-Positron Collider (BEPC) will provide an incomparably large database which enables people to achieve a high statistics for drawing a definite conclusion. The spin of $\eta_{c}$ is zero and decays into a $\Lambda \bar{\Lambda}$ pair, that is exactly the Bohm's version about the entangled EPR state, therefore should serve as an ideal probe for LHVT of QM. In the proposal, the polarizations of $\Lambda$ and $\bar{\Lambda}$ can be determined respectively by the momenta directions of $\pi^{+}$and $\pi^{-}$, and the relatively longer lifetime of $\Lambda(\bar{\Lambda})$ guarantees the space-like requirement. All of these set a favorable condition for directly testing the Bell inequality.

In the standard calculations of the quantum field theory, the transition matrix element of $\Lambda \rightarrow p \pi^{-}$reads $[4]$

$$
M_{\Lambda}=(4 \pi)^{1 / 2}\left(S+P \boldsymbol{\sigma}_{\Lambda} \cdot \mathbf{a}\right)
$$

where $\mathbf{a}$ is the unit vector of $\pi^{-}$in the CM frame of $\Lambda: \frac{\mathbf{P}_{\pi}^{\mathrm{cm}}}{\left|\mathbf{P}_{\pi}^{\mathrm{cm}}\right|}, S$ and $P$ are the $\mathrm{S}$ and $\mathrm{P}$ waveamplitudes respectively. $\bar{\Lambda} \rightarrow \bar{p} \pi^{+}$has a similar expression. Thus in the successive process $\Lambda \bar{\Lambda} \rightarrow p \pi^{-} \bar{p} \pi^{+}$the correlation between the momenta of the two pions can be converted into 
the correlation between polarizations of $\Lambda$ and $\bar{\Lambda}$.

$$
\begin{aligned}
I(\mathbf{a}, \mathbf{b}) & =\left\langle S\left|\left(M_{A} M_{B}\right)^{\dagger}\left(M_{A} M_{B}\right)\right| S\right\rangle \\
& =\left(\frac{|S|^{2}+|P|^{2}}{4 \pi}\right)^{2}\left(1+\alpha^{2} \mathbf{a} \cdot \mathbf{b}\right)
\end{aligned}
$$

where $\alpha \approx 0.642$.

Except the coefficient $\alpha^{2}$ and the normalization, this expression is the same as the correlation function for a system of two spin-1/2 particles given in the Bohm's version (5). If one can neglect the CP violation in the process, the expression can be understood as: polarization of $\Lambda$ is $\alpha \mathbf{b}$, while polarization of $\bar{\Lambda}$ is $\alpha \mathbf{a}$. Taking appropriate normalization, the correlation function can be written as

$$
R(\mathbf{a}, \mathbf{b})=1+\alpha^{2} \mathbf{a} \cdot \mathbf{b}
$$

and the corresponding Bell inequality is rephrased as

$$
|E(\mathbf{a}, \mathbf{b})-E(\mathbf{a}, \mathbf{c})| \leqslant 1+E(\mathbf{b}, \mathbf{c})
$$

where $E=\frac{1-R}{\alpha^{2}}$. Törnqvist defined a quantity $\cos \Theta_{a b} \equiv \mathbf{a} \cdot \mathbf{b}$, and by it one notices that the Bell inequality can be expressed as within a continuous region, variable $\Theta_{a b}$ satisfies an alternative inequality:

$$
\left|E\left(\Theta_{a b}\right)\right| \leqslant 1-2 \Theta_{a b} / \pi
$$

Obviously as shown above, by QFT, the correlation function $\left|E\left(\Theta_{a b}\right)\right|$ is linearly proportional to $\cos \Theta_{a b}$. In Fig. 1, for explicitness and clarity, we do not normalize the data, but keep the event number density where we choose each bin in the figure covering a region of 0.05 $\left(\cos \Theta_{a b}\right)$. According to Törnqvist's suggestion, the DM2 collaboration reported their preliminary result in 1985. Due to very poor statistics, they only presented the fitting results for $J / \psi \rightarrow \Lambda \bar{\Lambda} \rightarrow p \pi^{-} \bar{p} \pi^{+}$. Even though in this process $\Lambda$ and $\bar{\Lambda}$ also reside in an entangled state and the correlation functions are similar, there is an obvious difference from the $\eta_{c}$ case. Within error tolerance, their results do not show any obvious discrepancy from the QM prediction, on other aspect, one still cannot draw any conclusion from the analysis.

The BES III has begun operation and a great amount of $J / \psi$ and $\eta_{c}$ data has been accumulating, so the statistics is incomparably improved and one is confronting a valuable 


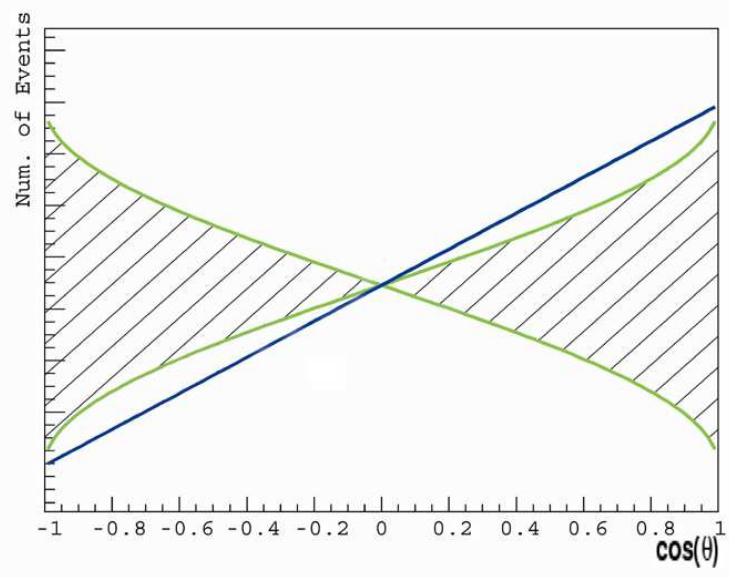

FIG. 1: the horizontal axis is $\cos \theta$ and the shadowed region is allowed by the Bell inequality, and the blue straightline is the QFT theoretical prediction

chance to make precise measurements on $\eta_{c} \rightarrow \Lambda \bar{\Lambda} \rightarrow p \pi^{-} \bar{p} \pi^{+}$, just based on this possibility, we will re-carry out a test on the LHVT at charm energy range. We are going to simulate the successive decay processes $J / \psi \rightarrow \gamma \eta_{c}, \eta_{c} \rightarrow \Lambda \bar{\Lambda} \rightarrow p \pi^{-} \bar{p} \pi^{+}$in terms of the MonteCarlo method and estimate the necessary event database for clearly distinguishing QM result from that obeys the Bell inequality. Even though for a real experimental setting, this simulation is not accurate enough, it still can provide a theoretical reference which may guide the experimentalists to design the experiment, or make decision if one can carry out the measurement with the considered luminosity and detection error tolerance at BES III.

\section{OUR METHOD}

\section{A. Random Sampling}

Generally, a complete Monte-Carlo simulation needs, 1. constructing the concerned random process, 2. taking random numbers which coincide with the probability function, 3 . estimating the errors and determining the confidence level of the result. 


\section{B. Goodness-of-fit Tests of $\chi^{2}$}

In this scheme, the quantity to be measured is the cosine of the angle between the two emitted pions. The statistical quantity

$$
\chi_{o b s}^{2}=\sum_{i=1}^{N} \frac{\left(n_{i}-n_{\text {theory }}\right)^{2}}{n_{i}}=\sum_{i=1}^{N} \frac{\left(n_{i}-n p_{0 i}\right)^{2}}{n_{i}},
$$

should approximately obey the $\chi^{2}(N-1)$ distribution. In the expression $N$ is the total number of the bins within the concerned physical region, $n_{i}$ is the experimental or M$\mathrm{C}$ estimated event number in the $i$-th bin, $n$ is the total event numbers (normalization: $\left.\sum n_{i}=n\right), n p_{0 i}$ is the theoretical event number and $p_{0 i}$ is the probability of event appearing in the i-th bin.

For a given Confidence Level (C.L.), $1-\alpha$, one can apply the $\chi^{2}(N-1)$ accumulation integration to determine the critical value of $\chi_{\alpha}^{2}(N-1)$ :

$$
\alpha=\int_{\chi_{\alpha}^{2}(N-1)}^{\infty} f(y ; N-1) d y .
$$

In above expression, $f(y ; N-1)$ is the probability density of $\chi^{2}(N-1)$ and the critical range is $\chi^{2}(N-1)>\chi_{\alpha}^{2}(N-1)$. Generally, if the theoretically evaluated value $\chi_{o b s}^{2}$ is greater than $\chi_{\alpha}^{2}(N-1)$, it indicates that data are not consistent with theory at the confidence level $1-\alpha$; whereas if the obtained $\chi_{o b s}^{2}$ is smaller than $\chi_{\alpha}^{2}(N-1)$, at $1-\alpha$ C.L, the data can be described by the theory to be tested.

\section{Simulation and results}

We are simulating the successive reactions $J / \psi \rightarrow \gamma \eta_{c}, \eta_{c} \rightarrow \Lambda \bar{\Lambda}, \Lambda \rightarrow p \pi^{-}, \bar{\Lambda} \rightarrow \bar{p} \pi^{+}$ step by step. Then taking into account of the Lorentz boost effects, we calculate the angle between the momenta of the two emitted pions which are measured in the center-of-mass reference frames of $\Lambda$ and $\bar{\Lambda}$ respectively.

In Table. I, one can note that $B R\left(J / \psi \rightarrow \eta_{c} \gamma\right) \times B R\left(\eta_{c} \rightarrow \Lambda \bar{\Lambda}\right) \times B R\left(\Lambda \rightarrow p \pi^{-}\right)^{2}=$ $5.5 \times 10^{-6}$, namely, for almost every $10^{6} \mathrm{~J} / \psi$ events, there would be 5.5 events corresponding to the expected reaction $J / \psi \rightarrow \eta_{c} \gamma \rightarrow \Lambda \bar{\Lambda} \gamma \rightarrow p \pi^{-} \bar{p} \pi^{+} \gamma$. Therefore we first carry out

our M-C simulation with $10^{7} \mathrm{~J} / \psi$ events, and then gradually increase the number of the produced $J / \psi$ events. According to the statistics, we would determine the necessary $J / \psi$ 
event number by which we can clearly distinguish the QFT result from the Bell inequality. In the following figures, the proportional errors are the reciprocal of the square-root of the event number in the corresponding bin, i.e. one standard deviation.

TABLE I: BR of Decay[10]

\begin{tabular}{c|c}
\hline channel & BR \\
\hline$J / \psi \rightarrow \eta_{c} \gamma$ & $(1.3 \pm 0.4) \%$ \\
\hline$\eta_{c} \rightarrow \Lambda \bar{\Lambda}$ & $(1.04 \pm 0.31) \times 10^{-3}$ \\
\hline$\Lambda \rightarrow p \pi^{-}$ & $(63.9 \pm 0.5) \%$ \\
\hline
\end{tabular}

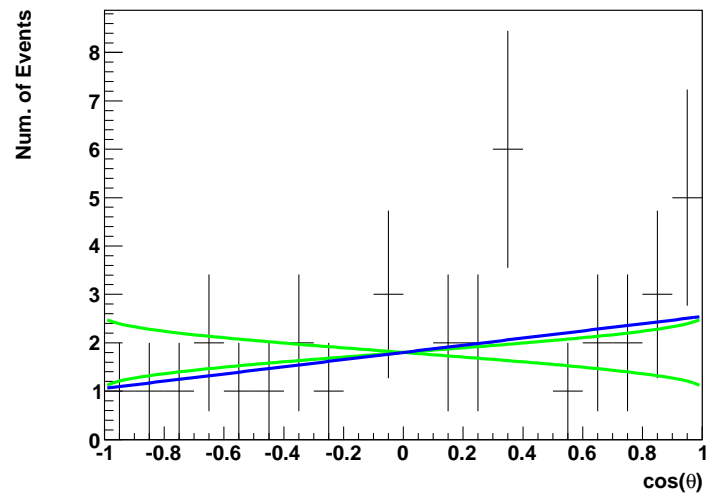

FIG. 2: $10^{7} \mathrm{~J} / \psi$ events, $\theta$ is the angle between $\pi^{-}$and $\pi^{+}$which are measured respectively in the center-of-mass reference frames of $\Lambda$ and $\bar{\Lambda}$. We divide the region of $\cos \theta[-1,+1]$ into 20 bins.

The results of the Monte-Carlo simulation are shown in Figs. 2, 3, 4, Then we employ the $\chi^{2}$ analysis to make the goodness-of-fit test to the boundary values of the QM prediction in comparison with the Bell inequality. The corresponding $\chi^{2}$ values are listed in Table II The employed equation is $\chi_{Q . M .}^{2}=\sum_{i=1}^{n}\left(n_{i}-n_{Q . M .}\right)^{2} / n_{i}, \chi_{B . I .}^{2}=\sum_{i=1}^{n}\left(n_{i}-n_{B . I .}\right)^{2} / n_{i}$.

In Fig. 2, one can observe that as $10^{7} \mathrm{~J} / \psi$ events are generated, there would only be 36 pion pairs, thus we can note a left-right asymmetry, but the Monte-Carlo results are not sufficient to test if QM are different from the Bell inequality. When the event number is increased to $10^{8}$, we obtain 395 pion pairs, and the results obviously incline into the prediction of QM (see Fig. 3). Even though the errorbars are still too large to clearly 


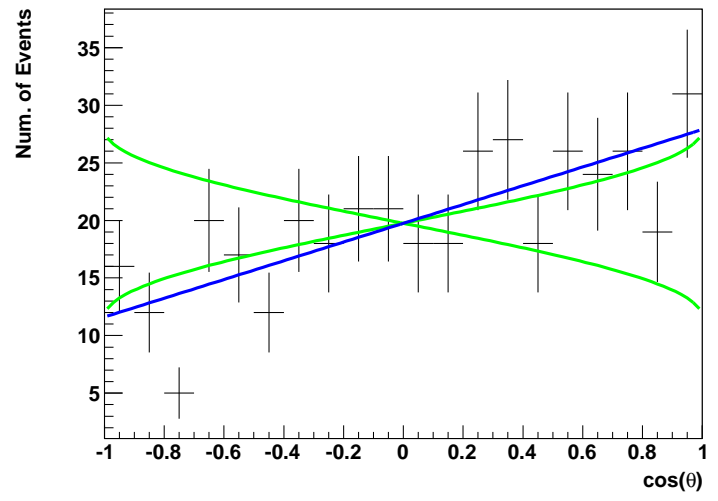

FIG. 3: $10^{8} \mathrm{~J} / \psi$ events, the other notations are the same as for Fig.2.

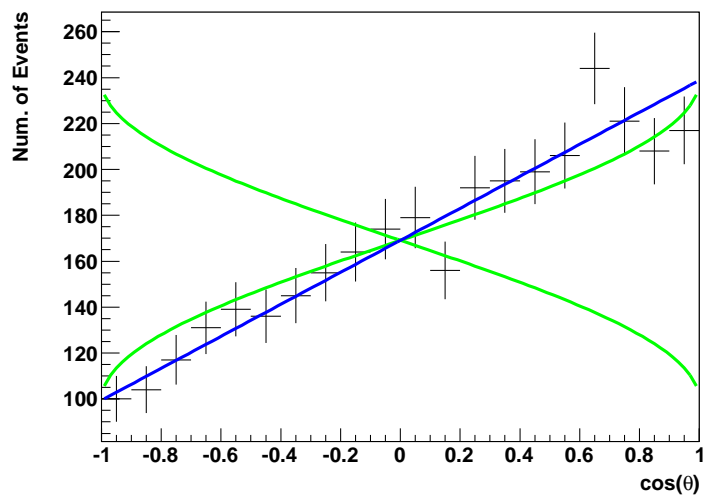

FIG. 4: $10^{9} \mathrm{~J} / \psi$ events, the other notations are the same as for Fig.2,

distinguish between the Bell inequality and the QM, by the $\chi^{2}$ best-fitting test, it is shown that $\chi_{Q . M .}^{2}<\chi_{B . I}^{2}$. and the $\chi^{2}$ of boundary value of the Bell inequality is larger than the QM value by $20 \%$. When the $J / \psi$ event number reaches $10^{9}$, we can generate 3382 pion pairs, the central values of the Monte-Carlo simulation distribute at the vicinity of the straightline predicted by the QM, Fig. 4, and the errorbars also are shortened to the length which is comparable with the deviation between the QM and Bell inequality. Then the goodness-of-fit is $\chi_{B . I .}^{2} / \chi_{Q . M .}^{2}=1.83$, i.e. the two theories result in remarkable distinction. It corresponds to $80 \%$ C.L. Numerical results are shown in the following table. For higher statistics (smaller statistical errors, oe higher C.L.) much more $J / \psi$ events are needed.

As a brief summary, it is possible to test the Bell inequality with a database of as large 
TABLE II: $\chi^{2}$ value of fit with different statistics

\begin{tabular}{|c|c|c|c|}
\hline statistics $(J / \psi)$ & $10^{7}$ & $10^{8}$ & $10^{9}$ \\
\hline$\chi_{Q . M .}^{2}$ & 7.20 & 27.44 & 13.07 \\
\hline$\chi_{\text {B.I. }}^{2}$ & 7.51 & 32.84 & 23.89 \\
\hline
\end{tabular}

as $10^{9} \mathrm{~J} / \psi$ events, but the accuracy is not sufficiently high as expected. Moreover, when detection efficiency is taken into account, this number must be at least multiplied by a factor of 10. Thus, for drawing a convincing conclusion, $10^{11} \mathrm{~J} / \psi$ events seem to be necessary, and it is probably beyond the reach of BES III for a few years unless its luminosity can be enhanced remarkably.

\section{DISCUSSION AND OUR NEW PROPOSAL}

In above section, we carefully investigate possibility of testing the Bell inequality at $e^{+} e^{-}$ collider via successive reactions $J / \psi \rightarrow \gamma \eta_{c}, \eta_{c} \rightarrow \Lambda \bar{\Lambda} \rightarrow p \pi^{-} \bar{p} \pi^{+}$. With our numerical simulation, we conclude that as the event number of $J / \psi$ reaches $10^{9}$, one can test the validity of the Bell inequality at $80 \%$ C.L., but a real test needs $10^{11} \mathrm{~J} / \psi$ events at least.

It is worth noticing that our above work does not make a judgement about validity of the LHVT, but assuming if Törnqvist's proposal really makes sense, how many $J / \psi$ events we need to realize the test i.e. to effectively distinguish the QM prediction from the region allowed by the Bell inequality. The analysis can be a reference for our BES colleagues.

Now let us turn to discuss a different issue which originates from critiques to the above experiment. According to those critiques, the reasonability of testing the LHVT with the successive decays $J / \psi \rightarrow \gamma \eta_{c}, \eta_{c} \rightarrow \Lambda \bar{\Lambda} \rightarrow p \pi^{-} \bar{p} \pi^{+}$proposed for by Törqvist is questionable. Namely, what the experiment is supposed to do is not the same as the fundamental idea of Bohm et al. based on analysis on LHVT.

The critiques are focusing on the key point: Can the aforementioned collider experiments testify if the Bell inequality holds? The critiques can be summarized into a few points.

1. The collider experiments are different from the optical experiments, namely they are not active measurements, but passive ones, in other words, we cannot control the 
momentum direction of the decay products, the pions, and then their polarizations.

2. Unlike proton and photon, in the collider experiments, the concerned particles are unstable and only leave tracks of a few cm, which are too short to let us make precise measurements on the spin polarizations of the involved particles.

3. The successive reaction such as $J / \psi \rightarrow \gamma \eta_{c}, \eta_{c} \rightarrow \Lambda \bar{\Lambda} \rightarrow p \pi^{-} \bar{p} \pi^{+}$, is very complicated, so that the measurements may not realize a concise test of the original Bell inequality.

4. There may exist a concept substitution[11]. In QM, different spin projections are noncommutative, and cannot be simultaneously measured. In collider experiments, such non-commutative quantities are replaced by commutative momentum components. That is different from the LHVT, namely there does not exist a subject to be measured which is related to the criterion of the EPR completeness. The reason is that as $\Lambda$ decays, in QM, it corresponds to a measurement and the momentum represents the result of measurement on the spin polarizations. Because the measurement induces a de-coherence, thus part of information is lost.

5. Finally, there is another logic problem. The spin-polarization correlation of $\Lambda$ and $\bar{\Lambda}$ is converted into the correlation between the pion momenta which are directly measured at collider as $1+\alpha^{2} \mathbf{a} \cdot \mathbf{b}$ where $\mathbf{a}$ and $\mathbf{b}$ are the directions of the $\pi^{\prime}$ s in the center-ofmass frames of their respective parent particles $(\Lambda$ or $\bar{\Lambda}$ )(comparing with eq.(8) where their spin polarizations are respectively $\alpha \mathbf{a}$ and $\alpha \mathbf{b}$ when the $\mathrm{CP}$ violation is ignored). However, in the original Bell inequality the quantity to be dealt with is $\mathbf{a} \cdot \mathbf{b}$ instead, thus the region covered by the Bell inequality is -1 to +1 while the direct measured momentum correlation is $1-\alpha^{2}$ to $1+\alpha^{2}$ which has an overlapping region with $[-1,+1]$. If one wants to check the distinction between the QM results with the Bell inequality, he must extract $\mathbf{a} \cdot \mathbf{b}$ from $1+\alpha^{2} \mathbf{a} \cdot \mathbf{b}$ which is derived from QFT (i.e. QM), thus he is confronting an embarrassing situation that he needs to admit validity of QM and uses it to testify QM. Generally, the result loses persuasiveness.

To overcome the problems, some authors [5] suggested to carry out the test in terms of the proton-proton secondary-scattering experiment. They planed to install a Stern-Gerlach apparatus to measure the spin-polarizations of proton. However, as indicated in the textbook 
of QM [8], because $\nabla \cdot \mathbf{B}=0$, the Lorentz force would smear out any observable effect as a non-uniform magnetic field applies.

Inspired by their ideas, we suggest to modify the method proposed by Törnqvist. Our proposal is following. One can install two Stern-Gerlach [12] apparatuses at two sides with flexible angles with respect to according to the electron-positron beams. The apparatus provides a non-uniform magnetic field which may decline trajectory of the neutral $\Lambda(\bar{\Lambda})$ due to its non-zero anomalous magnetic moment i.e. the force is proportional to $\frac{d}{\mathbf{n}}(-\boldsymbol{\mu} \cdot \mathbf{B})$ where $\boldsymbol{\mu}$ is the anomalous magnetic moment of $\Lambda, \mathbf{B}$ is a non-uniform external magnetic field and $\frac{d}{\mathbf{n}}$ is a directional derivative. Because $\Lambda$ is neutral, the Lorentz force does not apply, therefore one may expect to use the apparatus to directly measure the polarization of $\Lambda(\bar{\Lambda})$. The declination of trajectory of $\Lambda$ (here we use $\Lambda$ as an illustration) depends on its spin polarization. But one must first identify the particle flying into the Stern-Gerlach apparatus, i.e. to make sure it is $\Lambda$ or $\bar{\Lambda}$. It can be determined by its decay product, i.e. if its decay product is $p^{+} \pi^{-}$, it is $\Lambda$, otherwise is $\bar{\Lambda}$. Here one only needs the decay product to tag the decaying particle, but does not use it to do kinematic measurements.

The advantages are obvious that one can completely avoid the problems listed above. First, we turn a passive measurement into an active one, because we can independently adjust direction of each Stern-Gerlach apparatus, so that we can obtain spin-correlations among three independent directions as required by the original Bell inequality. This is in analog to the optical experiment and make real test on the Bell inequality.

One can install such an apparatus as illustrated in Fig. 5. particles 1 and 2 are produced in a spin singlet and later are separated by a space-like distance after a time interval $t$ and then fly into two Stern-Gerlach apparatuses with gradients of magnetic fields being $\mathbf{a}$ and $\mathbf{b}$ respectively. By their declinations, one can note their polarizations. It overcomes the flaws of original experimental setting.

There is no free lunch in the world, i.e. advantages also bring up more difficulties. Let us list a few problems which we can figure out at present.

1. The lifetime of $\Lambda$ is not very long, and as products of $\eta_{c}$, it can probably travel a few cm before decays, so it would be difficult to satisfy the space-like conditions. To enforcing the space-like condition, we need to install the two Stern-Gerlach apparatuses far enough. It means that we only choose the $\Lambda^{\prime}$ s which live longer, if so, the statistics 


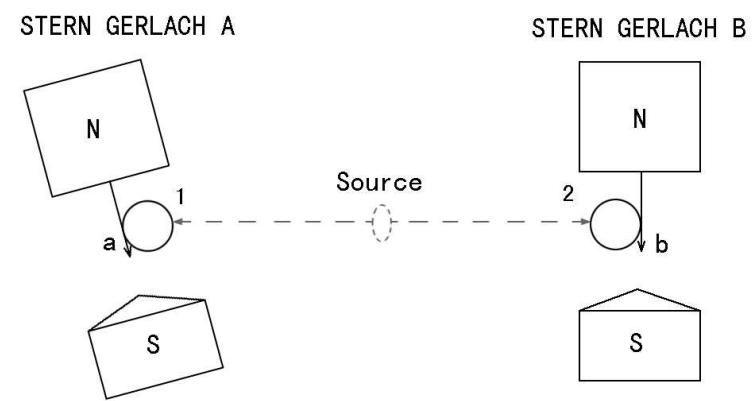

FIG. 5: The experimental setting with two Stern-Gerlach apparatuses

would be greatly decreased.

2. The magnetic field is not easily adjusted.

3. The decay products of $\Lambda$ are charged and they may leave very complicated trajectories in the non-uniform magnetic field and makes the event reconstruction very difficult. In other words, it is hard to determine the location of the production vertex.

4. Besides the above difficulties, one still confronts another serious problem. The magnetic fields in the detector and especially in the Stern-Gerlach apparatuses may flip the polarizations of $\Lambda$ and/or $\bar{\Lambda}$, thus the coherence between $\Lambda$ and $\bar{\Lambda}$ is spoiled[13]. Therefore the magnetic fields in the detector should not be too strong to cause such decoherence, but on other aspect they cannot be too weak, otherwise the declinations of the trajectories of $\Lambda$ and $\bar{\Lambda}$ are not detectable.

Even though all the problems are difficult, we believe that with rapid developments of high energy physics facilities and detection techniques they can be eventually solved and we will be able to testify the LHVT according to its original proposal.

\section{Acknowledgments}

This work was supported in part by the National Natural Science Foundation of China and the Special Grant for Ph.D programs of the Education Ministry of China. We are 
grateful for the helpful discussions with the members of the Bell Inequality group in China. One of us (Hao) would like to thank Dr. Y. L. Zhu for his directions on the Monte-Carlo simulations.

[1] A. Einstein, B. Podolski, N. Rosen, Phys. Rev. 47, 777(1935).

[2] D. Bohm, A. Aharonov, Phys. Rev. 108, 1070(1957).

[3] J. S. Bell, Physics 1, 195(1964).

[4] Nils A. Törnqvist et al., Found. Phys. 11, 171(1981).

[5] M. Lamehi-Rachti and W. Mitting, Phys. Rev. D14, 2543 (1976).

[6] A. Aspect, P. Grangier, and G. Roger, Phys. Rev. Lett. 49, 91 (1982); A.Aspect, J. Dalibard, and G. Roger, Phys. Rev. Lett. 49, 1804 (1982); G. Weihs et al., Phys. Rev. Lett. 81, 5039 (1998); Jian-Wei Pan, Dik Bouwmeester, Matthew Daniell, Harald Weinfurter, and Anton Zeilinger, Nature 403, 515 (2000); Andrew G. White, Daniel F.V. James, Philippe H. Eberhard, and Paul G. Kwiat, Phys. Rev. Lett. 83, 3103 (1999); Tao Yang, Qiang Zhang, Jun Zhang, Juan Yin, Zhi Zhao, Marek Zukowski, Zeng-Bing Chen, and Jian-Wei Pan, Phys. Lett. 95, 240406 (2005). D. Bouwmeester, J.-W. Pan, M. Daniell, H. Weinfurter. and A. Zeilinger, Phys. Rev. Lett. 82, 1345(1999); Zhi Zhao, Yu Ao Chen, An Ning Zhang, Tao Yang, Hans J. Briegel, and Jian Wei Pan, Nature 430, 54(2004).

[7] CPLEAR Collaboration, Phys. Lett. B 422, 339 (1998); M.H. Tixier et al. (the DM2 Collaboration, LAL Orsay, LPC, Clermont, Padova, Frascati), Presentation at: Conference on Microphysical Reality and Quantum Formalism, Urbino, Italy (1985).

[8] G. Baym, Lectures on Quantum Mechanics, The Benjamin/Cummings Pub.Co.INC., 1969, London.

[9] Reinhold A. Bertlmann, Lect. Notes Phys., 689, 1(2006); Schladming 2004, Quantum coherence, 1-45; C. Lupo, V. I. Man'ko, G. Marmo, J. Phys. A39,12515(2006); Yi-Bing Ding, Jun-li Li, Cong-Feng Qiao, HEP \& NP 31, 1097(2007) hep-ph/0702271.

[10] C. Amsler et al. (Particle Data Group), Phys. Lett. B667, 1 (2008).

[11] S.A. Abel, M. Dittmar, Herbert K. Dreiner, Phys. Lett. B280, 304(1992).

[12] W. Gerlach, O. Stern, Z.Phys. 9,349(1922) ;W. Gerlach, O. Stern, Z.Phys.8,110(1922).

[13] Wang Jin-Feng, Wang Yu-Ming, Li Xue-Qian, High Energy Physics and Nuclear Physics, 
29,14(2005). 